\title{
Identification of Potent and Selective Inhibitors of Fat Mass Obesity Associated Protein Using a Fragment-Merging Approach
}

Muthuraj Prakash, ${ }^{\dagger}$ Yukihiro Itoh, ${ }^{\dagger, * *}$ Yoshie Fujiwara, ${ }^{\S}$ Yukari Takahashi, ${ }^{\dagger}$ Yuri Takada, ${ }^{\dagger}$ Paolo Mellini, ${ }^{\dagger}$ Elghareeb E. Elboray, ${ }^{\dagger, / /}$ Mitsuhiro Terao, ${ }^{\ddagger}$ Yasunobu Yamashita, ${ }^{\dagger}$ Chika Yamamoto, ${ }^{\#}$ Takao Yamaguchi, ${ }^{\#}$ Masayuki Kotoku, ${ }^{\dagger}$ Yuki Kitao, ${ }^{\dagger}$ Ritesh Singh, ${ }^{\dagger, \nabla}$ Rohini Roy,,${ }^{\S, \circ}$ Satoshi Obika, ${ }^{\#}$ Makoto Oba, ${ }^{\dagger}$ Dan Ohtan

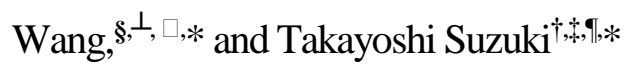

${ }^{\dagger}$ Graduate School of Medical Science, Kyoto Prefectural University of Medicine, 1-5 Shimogamohangi-cho, Sakyo-ku, Kyoto, 606-0823, Japan

${ }^{\sharp}$ SANKEN, Osaka University, Mihogaoka, Ibaraki-shi, Osaka 567-0047, Japan

§Institute for Integrated Cell-Material Sciences (iCeMS), Kyoto University, Yoshida-Honmachi, Sakyo-ku, Kyoto, 606-8501, Japan

"Chemistry Department, Faculty of Science, South Valley University, Qena, 83523, Egypt

\#Graduate School of Pharmaceutical Sciences, Osaka University, 1-6 Yamadaoka, Suita, Osaka 565-0871, Japan

${ }^{\nabla}$ Department of Chemistry, Central University of Rajasthan, NH-8, Bandar Sindri, Ajmer-305817, Rajasthan, India

${ }^{\circ}$ Graduate School of Biostudies, Kyoto University, Yoshida-Konoe cho, Sakyo-ku, Kyoto, 606-8501, Japan.

${ }^{\perp}$ Center for Biosystems Dynamics Research, RIKEN, 2-2-3 Minatojima-minamimachi, Chuo-ku, Kobe, Hyogo 650-0047, Japan

${ }^{\square}$ Wuya College of Innovation, Shenyang Pharmaceutical University, Shenyang, 110016, China

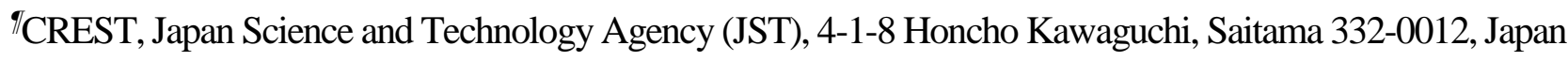


$\begin{array}{ll}\text { Supporting Figures } & \text { S3 }\end{array}$

Figure S1. In vitro enzyme assays of $\mathrm{Hz}(\mathbf{3})$ and rhein (5). $\quad$ S3

Figure S2. Isothermal titration calorimetry (ITC) analysis of compound 11b. S4

Figure S3. In vitro KDM4C inhibitory activites of 2,4-PDCA (2) and compound 11b. S5

Figure S4. Overview of compound 11b docked into FTO. S6

Figure S5. Steric hindrance of compounds 9a and 10a in the FTO catalytic site. S7

Figure S6. Viability of HL-60 cells treated with $\mathbf{2 9 b .}$ S8

$\begin{array}{ll}\text { Supporting Tables } & \text { S9 }\end{array}$

Table S1. Ligand efficiency of compounds 1-8, and $\mathbf{1 1}$ S9

$\begin{array}{ll}\text { Table S2. Primers for quantitative RT-PCR. S10 } & \text { S10 }\end{array}$

HPLC Charts of compounds 11a, 11b, and 29b. S11 
(a)

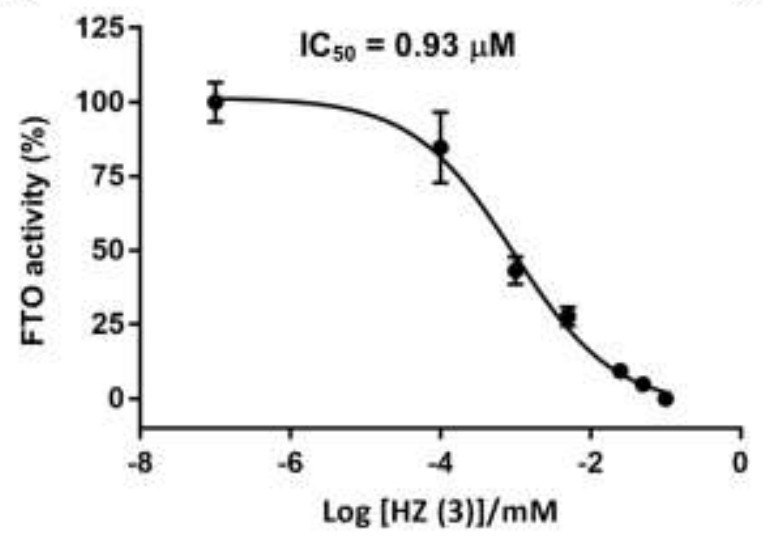

(b)

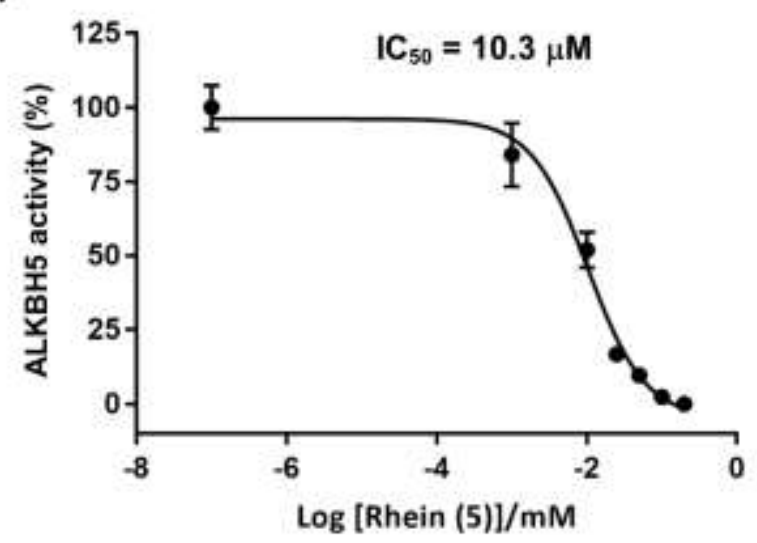

Figure S1. In vitro enzyme assays of $\mathrm{Hz}$ (3) and rhein (5). (a) Dose-response curves for FTO activity of $\mathrm{Hz}$ (3). (d) Dose-response curves for ALKBH5 activity of rhein (5). Error bars represent the mean standard deviation (SD) of at least three samples. 

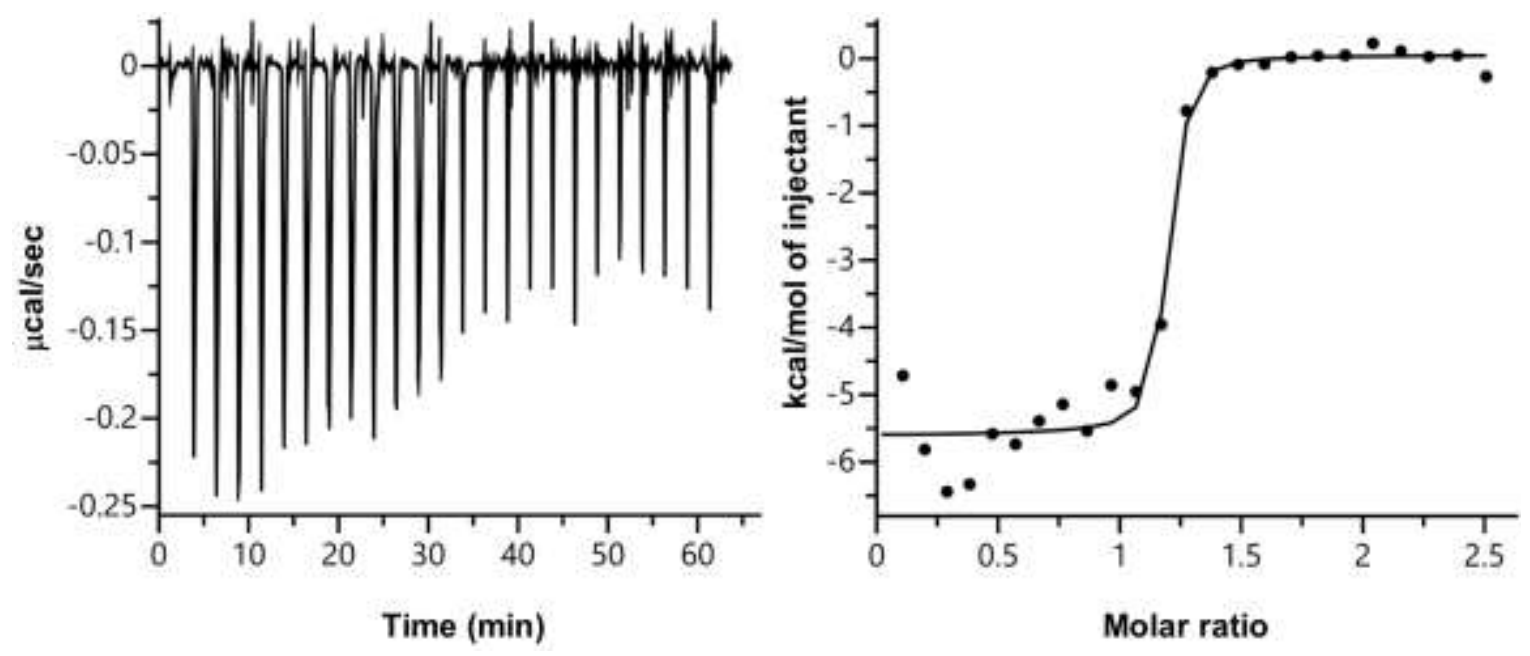

Figure S2. Isothermal titration calorimetry (ITC) analysis of compound $\mathbf{1 1 b}$. The titrations were conducted in $85 \%$ buffer (16 mM Tris $\mathrm{pH} 7.6,64 \mathrm{mM} \mathrm{NaCl}, 0.8 \mathrm{mM}$ dithiothreitol, and 10\% glycerol)/15\% DMSO. The sample chamber and syringe were filled with $30 \mu \mathrm{M}$ FTO and $180 \mu \mathrm{M} 11 \mathbf{b}$, respectively. The thermodynamic parameters are estimated as follows: $K_{\mathrm{d}}=28.0 \mathrm{nM}, \Delta H=-5.62 \mathrm{kcal} / \mathrm{mol}, \Delta G=-10.3 \mathrm{kcal} / \mathrm{mol},-\mathrm{T} \Delta S=-4.69$ $\mathrm{kcal} / \mathrm{mol}$. The ITC data was combined by two successive titrations with the same inhibitor solution by the ConCat ITC software (Malvern Panalytical). 


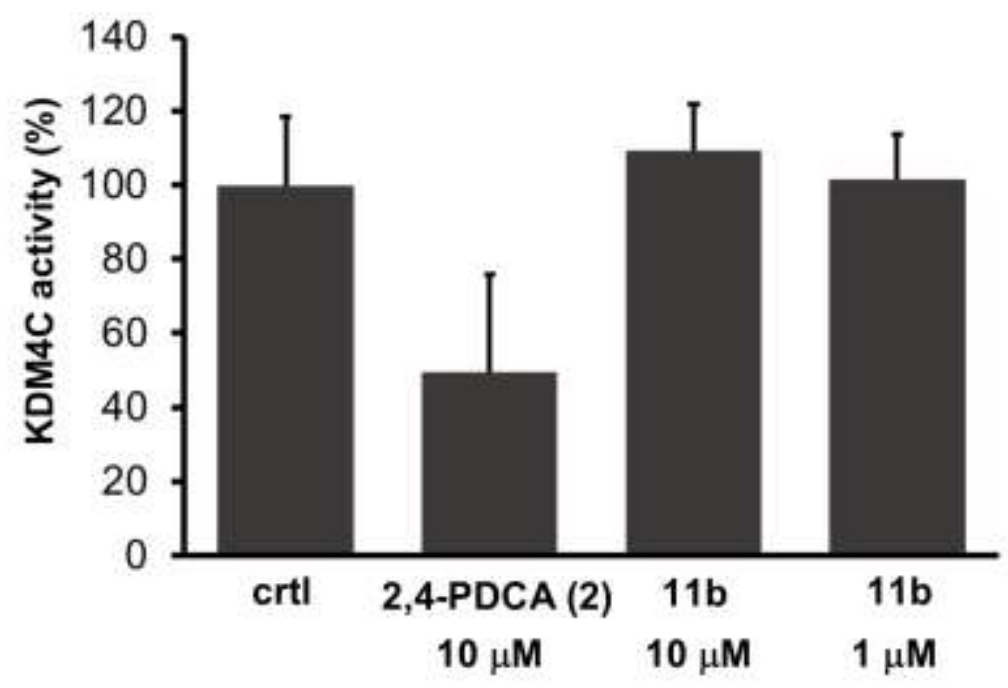

Figure S3. In vitro KDM4C inhibitory activities of 2,4-PDCA (2) and compound 11b. Bars indicate the mean and error bars represent the standard deviation (SD) of at least three samples. 


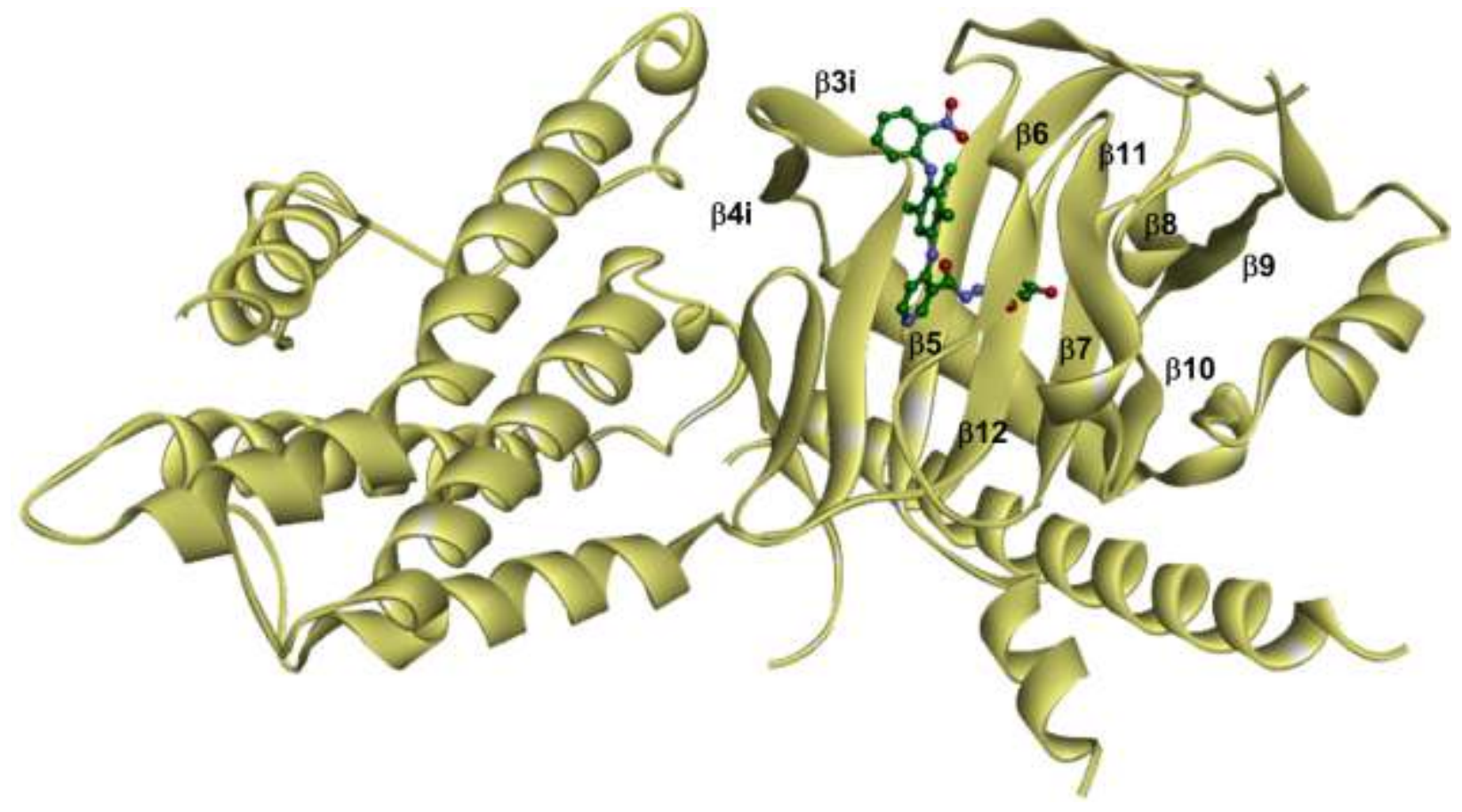

Figure S4. Overview of compound 11b docked into FTO. 
(a)
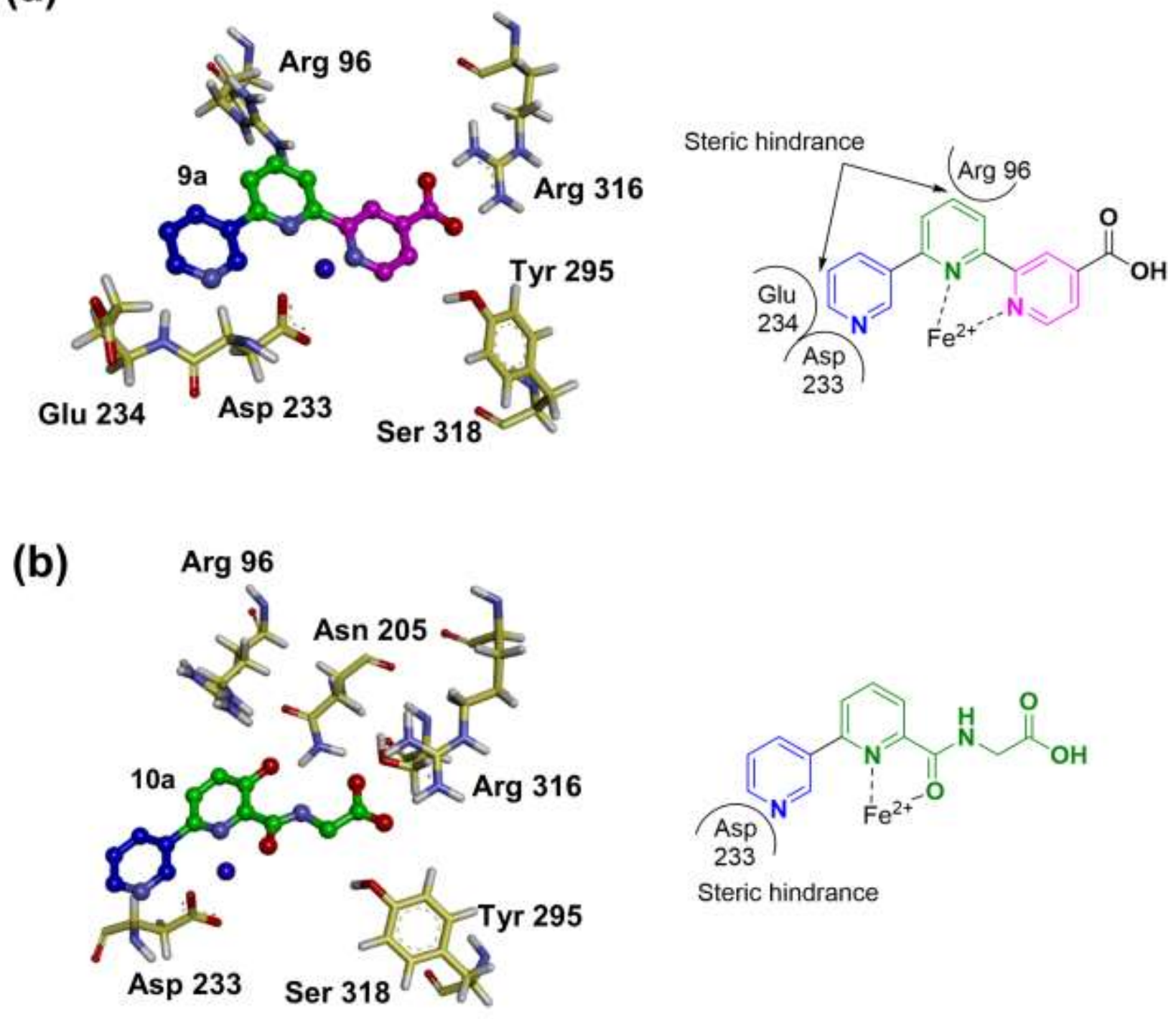

Figure S5. Steric hindrance of compounds (a) 9a and (b) 10a in the FTO catalytic site. The conformation models were prepared by superimposing compounds $9 \mathbf{a}$ and 10b on compounds 2 (PDB ID: 4IE0) and $\mathbf{1}$ (PDB ID: 4IE5) in the catalytic site, respectively. 


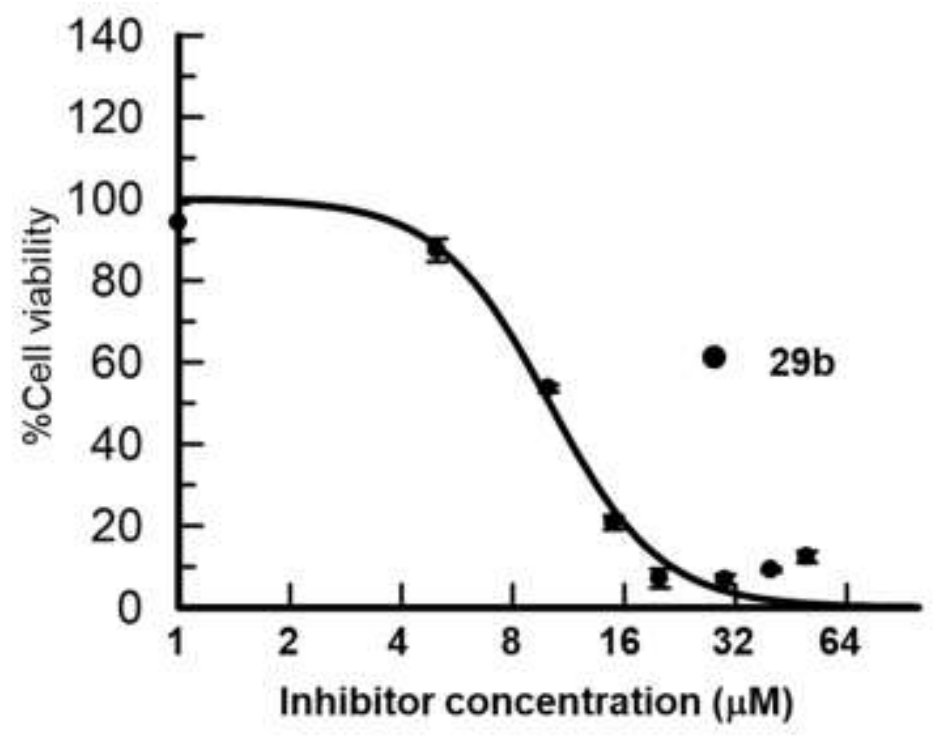

Figure S6. Viability of HL-60 cells treated with 29b. 
Table S1. Ligand efficacy of compounds $1-8$ and $11 .^{a}$

\begin{tabular}{cccc}
\hline compound & FTO IC $50(\mu \mathrm{M})$ & heavy atom number & Ligand efficiency \\
\hline $\mathbf{1}$ & 15 & 14 & 0.48 \\
$\mathbf{2}$ & 8.3 & 12 & 0.59 \\
$\mathbf{4}$ & 0.93 & 24 & 0.35 \\
$\mathbf{5}$ & 8 & 19 & 0.38 \\
$\mathbf{6}$ & 9.0 & 21 & 0.34 \\
$\mathbf{7}$ & 0.143 & 30 & 0.32 \\
$\mathbf{8}$ & 0.713 & 28 & 0.31 \\
$\mathbf{1 1 a}$ & 3.39 & 18 & 0.43 \\
$\mathbf{1 1 b}$ & 0.57 & 36 & 0.24 \\
\hline
\end{tabular}

${ }^{a} \mathrm{LE}$ values were calculated using the following equation: $\mathrm{LE}=(\Delta G) / \mathrm{N}=1.4 \times\left(-\log \mathrm{IC}_{50}\right) / \mathrm{N}$. 
Table S2. Primers for quantitative RT-PCR.

\begin{tabular}{ccc}
\hline Genes & Forward primers (5'-3') & Reverse primers (5'-3') \\
\hline MYC & GTCAAGAGGCGAACACACAAC & TTGGACGGACAGGATGTATGC \\
RARA & GCATGTCCAAGGAGTCTGTGA & CGTCAGCGTGTAGCTCTCA \\
28S rRNA & TGTCGGCTCTTCCTATCATTGT & ACCCAGCTCACGTTCCCTATTA \\
ACTIN & CACTCTTCCAGCCTTCCTTC & GTACAGGTCTTTGCGGATGT \\
\hline
\end{tabular}


HPLC (compound 11a)

mAU

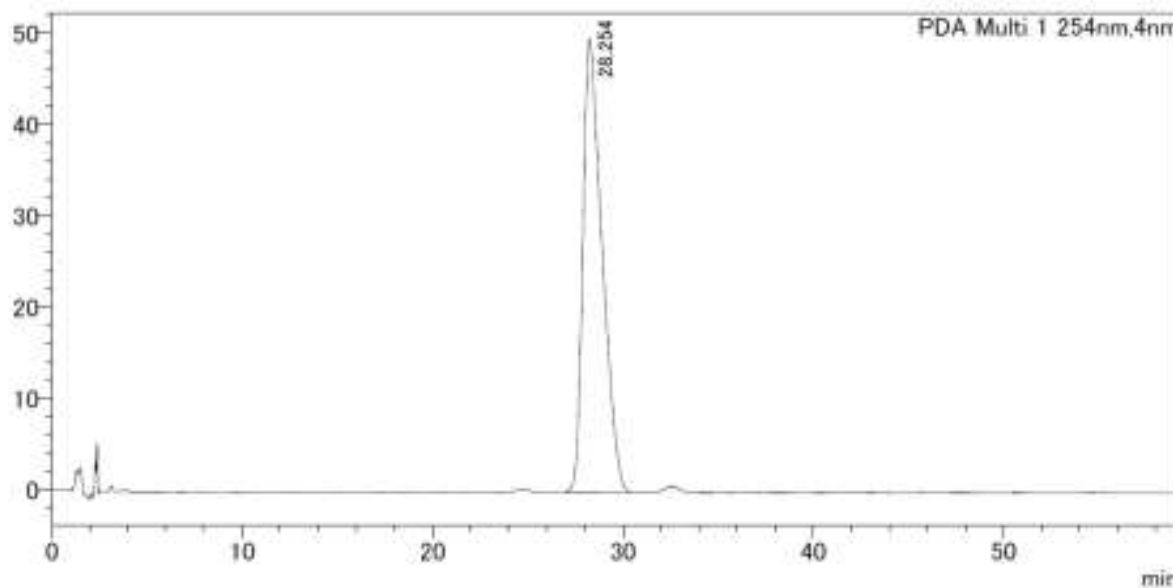

\begin{tabular}{cc}
\hline $\begin{array}{c}\text { RT } \\
(\mathrm{min})\end{array}$ & $\begin{array}{c}\% \\
\text { area }\end{array}$ \\
\hline 24.787 & 0.543 \\
28.254 & 98.661 \\
32.605 & 0.796 \\
\hline
\end{tabular}

HPLC (compound 11b)

mAL

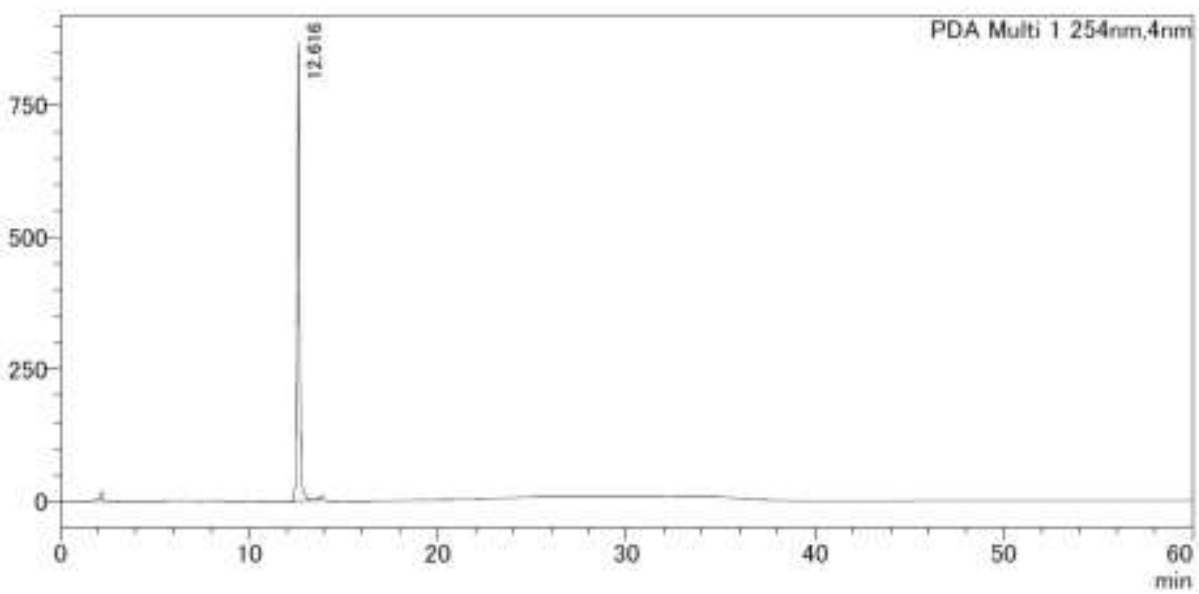

\begin{tabular}{cc}
\hline $\begin{array}{c}\mathrm{RT} \\
(\mathrm{min})\end{array}$ & $\begin{array}{c}\% \\
\text { area }\end{array}$ \\
\hline 12.811 & 0.109 \\
12.616 & 96.722 \\
13.409 & 0.041 \\
13.621 & 0.252 \\
13.855 & 0.986 \\
14.520 & 0.029 \\
23.969 & 0.018 \\
\hline
\end{tabular}

HPLC (compound 29b)

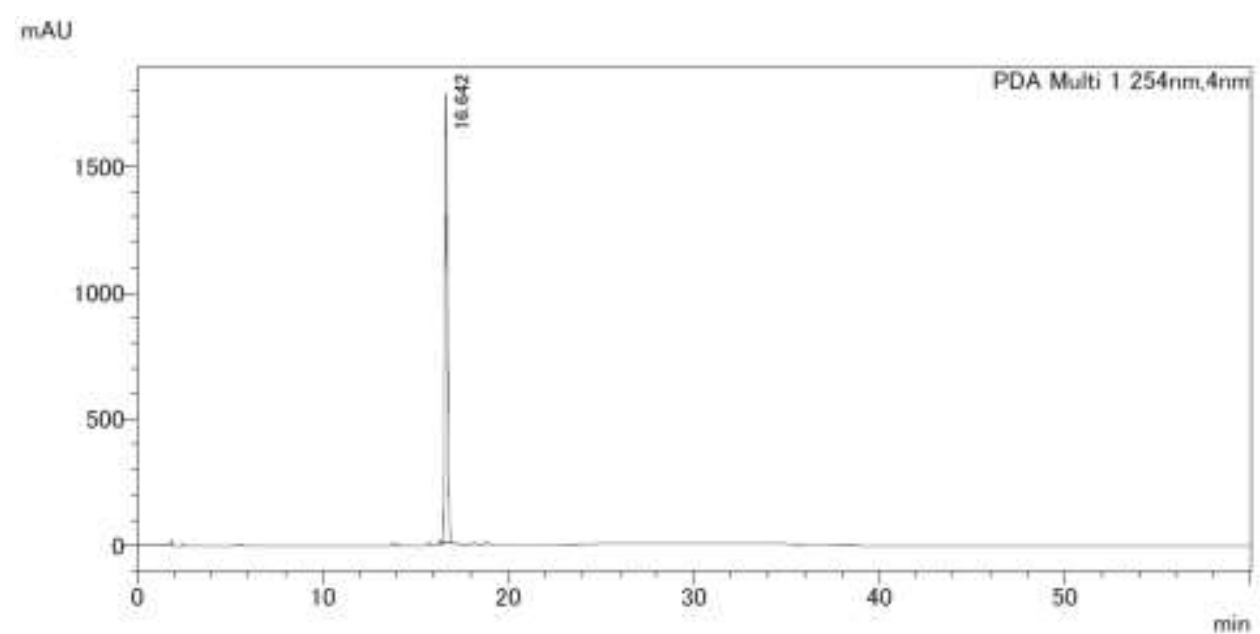

\begin{tabular}{cc}
\hline $\begin{array}{c}\mathrm{RT} \\
(\mathrm{min})\end{array}$ & $\begin{array}{c}\% \\
\text { area }\end{array}$ \\
\hline 13.769 & 0.455 \\
13.929 & 0.217 \\
15.722 & 0.726 \\
16.082 & 0.060 \\
16.303 & 0.834 \\
16.642 & 95.652 \\
17.046 & 0.213 \\
17.357 & 0.124 \\
17.741 & 0.204 \\
17.980 & 0.119 \\
18.156 & 0.150 \\
18,260 & 0.260 \\
18.433 & 0.085 \\
18.826 & 0.461 \\
\hline
\end{tabular}

